\title{
Fractional Order Two-Temperature Dual-Phase-Lag Thermoelasticity with Variable Thermal Conductivity
}

\author{
Sudip Mondal, ${ }^{1}$ Sadek Hossain Mallik, ${ }^{2}$ and M. Kanoria ${ }^{3}$ \\ ${ }^{1}$ Bhatkunda High School, Burdwan 713153, India \\ ${ }^{2}$ Department of Mathematics, Aliah University, Kolkata 700091, India \\ ${ }^{3}$ Department of Applied Mathematics, University of Calcutta, Kolkata 700009, India
}

Correspondence should be addressed to M. Kanoria; k_mri@yahoo.com

Received 10 May 2014; Revised 4 August 2014; Accepted 4 August 2014; Published 29 October 2014

Academic Editor: Abdelouahed Tounsi

Copyright (C) 2014 Sudip Mondal et al. This is an open access article distributed under the Creative Commons Attribution License, which permits unrestricted use, distribution, and reproduction in any medium, provided the original work is properly cited.

\begin{abstract}
A new theory of two-temperature generalized thermoelasticity is constructed in the context of a new consideration of dualphase-lag heat conduction with fractional orders. The theory is then adopted to study thermoelastic interaction in an isotropic homogenous semi-infinite generalized thermoelastic solids with variable thermal conductivity whose boundary is subjected to thermal and mechanical loading. The basic equations of the problem have been written in the form of a vector-matrix differential equation in the Laplace transform domain, which is then solved by using a state space approach. The inversion of Laplace transforms is computed numerically using the method of Fourier series expansion technique. The numerical estimates of the quantities of physical interest are obtained and depicted graphically. Some comparisons of the thermophysical quantities are shown in figures to study the effects of the variable thermal conductivity, temperature discrepancy, and the fractional order parameter.
\end{abstract}

\section{Introduction}

During the last five decades, nonclassical thermoelasticity theories involving hyperbolic type heat transport equations admitting finite speed of thermal signals have been formulated. According to these theories, heat propagation is to be viewed as a wave phenomenon rather than a diffusion phenomenon.

In order to overcome the paradox of an infinite speed of thermal wave inherent in CTE and CCTE (classical coupled theory of thermoelasticity), efforts were made to modify coupled thermoelasticity, on different grounds, to obtain a wavetype heat conduction equation by different researchers. Lord and Shulman [1] formulated the generalized thermoelasticity theory introducing one relaxation time in Fourier's law of heat conduction equation and thus transforming the heat conduction equation into a hyperbolic type.

Green and Lindsay [2] used the theory of two different relaxation times in the constitutive relations for the stress tensor and the entropy equation. Later Green and Naghdi [35] have proposed three models, labeled as types I, II, and III.
When they are linearized, type I is the same as the classical heat equation (based on Fourier's law) whereas the linearized versions of type-II and type-III theories permit propagation of thermal waves at finite speed. The entropy flux vector in type-II (i.e., thermoelasticity without energy dissipation) and type-III (i.e., thermoelasticity with energy dissipation) models is determined in terms of the potential that also determines stresses. When Fourier conductivity is dominant, then the temperature equation reduces to classical Fourier's law of heat conduction and when the effect of conductivity is negligible, then the equation has undamped thermal wave solutions without energy dissipation.

Tzou [6] introduced two-phase-lag models to both the heat flux vector and the temperature gradient. According to this model, classical Fourier's law $\vec{q}=-K \vec{\nabla} \theta$ has been replaced by $\vec{q}\left(P, t+\tau_{q}\right)=-K \nabla \theta\left(P, t+\tau_{T}\right)$, where the temperature gradient $\vec{\nabla} \theta$ at a point $P$ of the material at time $t+\tau_{T}$ corresponds to the heat flux vector $\vec{q}$ at the same point at time $t+\tau_{q}$. Here $K$ is the thermal conductivity of the material. The delay time $\tau_{T}$ is interpreted as that caused by the 
microstructural interactions and is called the phase-lag of the temperature gradient. The other delay time $\tau_{q}$ is interpreted as the relaxation time due to the fast transient effects of thermal inertia and is called the phase-lag of the heat flux. The case $\tau_{q}=\tau_{T}=0$ corresponds to classical Fourier's law. If $\tau_{q}=$ $\tau$ and $\tau_{T}=0$, Tzou refers to the model as single-phaselag model. Recently Several researchers have attempted to solve their problems on the basis of the theory of dual-phaselag model. Roychoudhuri [7] has studied one-dimensional thermoelastic wave propagation in an elastic half-space in the context of dual-phase-lag model. The exponential stability [8] and condition of the delay parameters in the dual-phase-lag theory [9] under this model have been studied by Quintanilla. Wang et al. [10, 11] have studied the well-posedness and solution structure of the dual-phase-lag heat conduction equation. Wang and Mingtian [12] have studied the thermal oscillation and resonance in dual-phase-lag heat conduction equation. Ailawalia and Budhiraja have studied a problem of dual-phase-lag model with internal heat source [13].

Gurtin and Williams $[14,15]$ have suggested that there is no a priori ground for assuming that the second law of thermodynamics for continuous bodies involves only a single temperature; that is, it is more logical to assume a second law in which the entropy contribution due to heat conduction is governed by one temperature, that of the heat supply by another.

Chen and Gurtin [16] and Chen et al. [17, 18] have formulated a theory of heat conduction in deformable bodies, which depends on two distinct temperatures: the conductive temperature $\phi$ and the thermodynamic temperature $\theta$. For time-independent situations, the differences between these two temperatures are proportional to the heat supply, and in the absence of any heat supply, the two temperatures are identical [18]. For time-dependent problems, however, and for wave propagation problems in particular, the two temperatures are, in general, different, independent of the presence of a heat supply. The key element that sets the twotemperature thermoelasticity (2TT) apart from the classical theory of thermoelasticity (CTE) is the material parameter $a(\geq) 0$, called the temperature discrepancy [18]. Specifically, if $a=0$, then $\phi=\theta$ and the field equations of the 2TT reduce to those of CTE.

The linearized version of the two-temperature theory (2TT) has been studied by many authors. Warren and Chen [19] have investigated the wave propagation in the two-temperature theory of thermoelasticity. Lesan [20] has established uniqueness and reciprocity theorems for the 2TT. Puri and Jordan [21] have studied propagation of plane waves under the 2TT. The existence, structural stability, and spatial behavior of the solution in 2TT have been discussed by Quintanilla [22].

It should be pointed out that both CTE and 2TT suffer from the so-called paradox of heat conduction, that is, the prediction that a thermal disturbance at some point in a body is felt instantly, but unequally, throughout the body. Mondal et al. $[23,24]$ have studied problems on two-temperature Green Naghdi III and dual-phase-lag model with variable thermal conductivity. Pal et al. [25], Islam et al. [26], Das and Kanoria [27], and Banik and Kanoria [28] have studied on two-temperature generalized thermoelasticity. Kumar et al. $[29,30]$ have established variational and reciprocal principles and some theorems in two-temperature generalized thermoelasticity. Recently Ailawalia et al. have solved a dynamic problem on Green-Naghdi (Type III) half-space with twotemperature [31] theory.

In this work, we have studied the thermoelastic stress, strain, displacement, conductive temperature, and the thermodynamic temperature in an infinite, isotropic, homogeneous elastic half-space under thermal shock using twotemperature dual-phase-lag generalized thermoelasticity in the context of fractional heat conduction equation. The governing equations of two-temperature generalized thermoelasticity theory are formed in the Laplace transform domain which are then solved by state space approach. The inversion of the transformed solutions is carried out numerically, applying a method based on a Fourier series expansion technique [32]. Finally, numerical estimate for different thermophysical quantities are obtained for copper materials. A complete and comprehensive analysis of the results is presented and the effects of fractional order parameter, two-type temperature, and variable thermal conductivity are discussed.

\section{Development of Fractional Order Theory}

Differential equations of fractional order have been the focus of many studies due to their frequent appearance in various applications in fluid mechanics, viscoelasticity, biology, physics, and engineering. The most important advantage of using fractional differential equations in these and other applications is their nonlocal property. It is well-known that the integer order differential operator is a local operator but the fractional order differential operator is nonlocal. This means that the next state of a system depends not only upon its current state, but also upon all of its historical states. This is more realistic, and this is one reason why fractional calculus has become more and more popular [33-35].

Fractional calculus has been used successfully to modify many existing models of physical processes. One can state that the whole theory of fractional derivatives and integrals was established in the second half of the nineteenth century. The first application of fractional derivatives was given by Abel who applied fractional calculus in the solution of an integral equation that arises in the formulation of the tautochrone problem. The generalization of the concept of derivative and integral to a noninteger order has been subjected to several approaches, and some various alternative definitions of fractional derivatives have appeared [36-39]. In the last few years, fractional calculus has been applied successfully in various areas to modify many existing models of physical processes, for example, chemistry, biology, modeling and identification, electronics, wave propagation, and viscoelasticity. One can refer to Podlubny [35] for a survey of applications of fractional calculus.

Youssef [40] introduced the formula of heat conduction given by

$$
q_{i}+\tau_{0} \frac{\partial q_{i}}{\partial t}=-K I^{\xi-1} \theta_{, i}, \quad 0<\xi \leq 2,
$$


where the notation $I^{\xi}$ is the Riemann-Liouville fractional integral, introduced as a natural generalization of the well-known $n$-fold repeated integral $I^{n} f(t)$ written in a convolution-type form as in [41] which is written as follows:

$$
\begin{aligned}
I^{n} f(t) & =\frac{1}{\Gamma(n)} \int_{0}^{t}(t-\tau)^{n-1} f(\tau) d \tau, \quad 0<n \leq 2, \\
& =f(t), \quad n=0
\end{aligned}
$$

where $\Gamma(n)$ is the Gamma function. A uniqueness theorem also has been proved. Sur and Kanoria have employed the theory to study problems on functionally graded [42] and viscoelastic material [43].

Ezzat and El-Karamany $[44,45]$ established a new model of fractional heat conduction equation by using the new Taylor series expansion of time-fractional order, developed by Jumarie [46] as

$$
q_{i}+\frac{\tau_{0}^{\xi}}{\xi !} \frac{\partial^{\xi} q_{i}}{\partial t^{\xi}}=-K \theta_{, i}, \quad 0<\xi \leq 1 .
$$

El-Karamany and Ezzat [47] introduced two general models of fractional heat conduction law for a nonhomogeneous anisotropic elastic solid. Uniqueness and reciprocal theorems are proved, and the convolutional variational principle is established and used to prove a uniqueness theorem with no restriction on the elasticity or thermal conductivity tensors except symmetry conditions. For fractional thermoelasticity not involving two-temperature, El-Karamany and Ezzat [48] established the uniqueness, reciprocal theorems and convolution variational principle. For two-temperature theory the formula of heat conduction has been replaced by

$$
q_{i}+\tau_{0} \frac{\partial q_{i}}{\partial t}=-K I^{\xi-1} \phi_{,}, \quad 0<\xi \leq 2,
$$

where $\phi$ is the conductive temperature. Two-temperature fractional order thermoelasticity problem of LS and Green Naghdi models (types II and III) have been solved by Sur and Kanoria [49].

Several researchers have solved different problems [5054] using fractional order generalized thermoelasticity theory. More detailed discussion on the subject is available in the books of Hetnarski and Eslami [55], Eslami et al. [56], and Ignaczak and Ostoja-Starzewski [57].

\section{Basic Formulation}

The constitutive equations are

$$
\sigma_{i j}=2 \mu e_{i j}+(\lambda e-\gamma \theta) \delta_{i j}, \quad i, j=1,2,3,
$$

where $e_{i j}=(1 / 2)\left(u_{i, j}+u_{j, i}\right)$ and $e=e_{k k}$.

In the context of two-temperature dual-phase-lag (2TDPL) generalized thermoelasticity theory, the equation of motion in the absence of body forces and the heat conduction equation in absence of heat sources for a linearly isotropic generalized thermoelastic solid based on the theory of fractional integral are, respectively, given by [58]

$$
\begin{gathered}
\rho \ddot{u}_{i}=(\lambda+\mu) u_{j, j i}+\mu u_{i, j j}-\gamma \theta_{, i}, \quad i, j=1,2,3, \\
{\left[K I^{\xi-1}\left(1+\tau_{T} \frac{\partial}{\partial t}+\frac{\tau_{T}^{2}}{2} \frac{\partial^{2}}{\partial t^{2}}\right) \phi_{, i}\right]_{, i}} \\
=\left(1+\tau_{q} \frac{\partial}{\partial t}+\frac{\tau_{q}^{2}}{2} \frac{\partial^{2}}{\partial t^{2}}\right)\left(\frac{K}{\kappa} \dot{\theta}+\gamma T_{0} \dot{e}\right),
\end{gathered}
$$

where $\rho$ is the density, $\lambda$ and $\mu$ are Lamé's constants, $K$ is thermal conductivity, $\gamma=(3 \lambda+2 \mu) \alpha_{t}$, $\alpha_{t}$ being the coefficient of linear thermal expansion, $T_{0}$ is the reference temperature, and $\kappa=K / \rho c_{E}, c_{E}$ being the specific heat at constant strain.

The relation between conductive temperature $(\phi)$ and thermodynamic temperature $(\theta)$ is given by

$$
\phi-\theta=a \phi_{, i i} \quad i=1,2,3,
$$

where $a(\geq 0)$ is the two-temperature parameter, called temperature discrepancy.

We will consider the thermal conductivity as a linear function of thermodynamical temperature as follows [59]:

$$
K(\theta)=K_{0}\left[1+K_{1} \theta\right],
$$

where $K_{0}$ is a constant which is equal to the thermal conductivity of the material when it does not depend on thermodynamical temperature $(\theta)$ and $K_{1}$ is a nonpositive small parameter.

Substituting from (8) into (9), we get

$$
\begin{aligned}
K(\theta) & =K_{0}\left[1+K_{1} \phi-a K_{1} \phi_{, i i}\right] \\
\text { or } \quad K(\theta) & =K_{0}\left[1+K_{1} \phi\right]-a K_{0} K_{1} \phi_{, i i} \\
\text { or } \quad K(\theta) & =K(\phi)-a K_{0} K_{1} \phi_{, i i} .
\end{aligned}
$$

We now use the following mapping [59]:

$$
\begin{aligned}
& \tilde{\phi}=\frac{1}{K_{0}} \int_{0}^{\phi} K(\tau) d \tau, \\
& \widetilde{\theta}=\frac{1}{K_{0}} \int_{0}^{\theta} K(\tau) d \tau .
\end{aligned}
$$

Differentiating (11) with respect to $x_{i}$, we get

$$
K_{0} \widetilde{\phi}_{, i}=K(\phi) \phi_{, i} .
$$

Differentiating again the above equation, we obtain

$$
K_{0} \tilde{\phi}_{, i i}=\left[K(\phi) \phi_{, i}\right]_{, i} .
$$

Differentiating (12) with respect to $x_{i}$, we get

$$
K_{0} \widetilde{\theta}_{, i}=K(\theta) \theta_{, i} .
$$

Differentiating (12) with respect to $t$, we get

$$
K_{0} \dot{\tilde{\theta}}=K(\theta) \dot{\theta} .
$$


Substituting from (14) and (16) into (7), we obtain

$$
\begin{aligned}
I^{\xi-1} & \left(1+\tau_{T} \frac{\partial}{\partial t}+\frac{\tau_{T}^{2}}{2} \frac{\partial^{2}}{\partial t^{2}}\right) \tilde{\phi}_{, i i} \\
= & \left(1+\tau_{q} \frac{\partial}{\partial t}+\frac{\tau_{q}^{2}}{2} \frac{\partial^{2}}{\partial t^{2}}\right)\left(\frac{\dot{\tilde{\theta}}}{\kappa}+\frac{\gamma T_{0}}{K_{0}} \dot{e}\right) \\
& +\frac{a K_{0} K_{1}}{2}\left[I^{\xi-1}\left(1+\tau_{T} \frac{\partial}{\partial t}+\frac{\tau_{T}^{2}}{2} \frac{\partial^{2}}{\partial t^{2}}\right)\left(\phi_{, i}\right)^{2}\right] .
\end{aligned}
$$

Neglecting the last term on right hand side of the above equation due to nonlinearity, we get

$$
\begin{aligned}
& I^{\xi-1}\left(1+\tau_{T} \frac{\partial}{\partial t}+\frac{\tau_{T}^{2}}{2} \frac{\partial^{2}}{\partial t^{2}}\right) \tilde{\phi}_{, i i} \\
& \quad=\left(1+\tau_{q} \frac{\partial}{\partial t}+\frac{\tau_{q}^{2}}{2} \frac{\partial^{2}}{\partial t^{2}}\right)\left(\frac{\dot{\tilde{\theta}}}{\kappa}+\frac{\gamma T_{0}}{K_{0}} \dot{e}\right) .
\end{aligned}
$$

Using (15) in (6), we get

$$
\rho \ddot{u}_{i}=(\lambda+\mu) u_{j, j i}+\mu u_{i, j j}-\frac{\gamma K_{0}}{K(\theta)} \tilde{\theta}_{, i} .
$$

For linearity we can approximate last equation to the following form:

$$
\rho \ddot{u}_{i}=(\lambda+\mu) u_{j, j i}+\mu u_{i, j j}-\gamma \widetilde{\theta}_{, i} \text {. }
$$

Now to transform (8) by using (11) and (12), we first replace the dummy variable $i$ with $k$ and then differentiating with respect to $x_{i}$ and finally multiplying by $K(\theta)$ we get

$$
K(\theta) \phi_{, i}-K(\theta) \theta_{, i}=a K(\theta) \phi_{, k k i}, \quad i, k=1,2,3 .
$$

Now substituting from (10) into (21) we have

$$
\begin{aligned}
& K(\phi) \phi_{, i}-K(\theta) \theta_{, i} \\
& \quad=a K(\phi) \phi_{, k k i}+\frac{a K_{0} K_{1}}{2}\left[\left\{\left(\phi_{, i}\right)^{2}\right\}_{, i}-a\left\{\left(\phi_{, k k}\right)^{2}\right\}_{, i}\right] .
\end{aligned}
$$

For linearity we can approximate the last equation as

$$
K(\phi) \phi_{, i}-K(\theta) \theta_{, i}=a K(\phi) \phi_{, k k i} .
$$

Retaining only the linear terms, (14) can be written as

$$
K_{0} \tilde{\phi}_{, k k i}=K(\phi) \phi_{, k k i}
$$

Now substituting from (13), (15), and (24) into (23) we have

$$
\tilde{\phi}_{, i}-\tilde{\theta}_{, i}=a \tilde{\phi}_{, k k i}, \quad i, k=1,2,3 \text {. }
$$

Now integrating with respect to $x_{i}$ we get

$$
\tilde{\phi}-\tilde{\theta}=a \tilde{\phi}_{, i i}, \quad i=1,2,3 .
$$

\section{Formulation of the Problem}

We consider a half space $(0 \leq x<\infty)$ with $x$-axis pointing to the medium. This half-space is subjected to thermal and mechanical loads on the bounding plane $(x=0)$ that depends on the time $t$ and is linearly quiescent. We will consider onedimensional thermoelastic deformation of the body so that the displacement components can be taken in the following form:

$$
\left(u_{x}, u_{y}, u_{z}\right)=(u(x, t), 0,0)
$$

The strain displacement relation is

$$
e_{x x}=\frac{\partial u}{\partial x}
$$

and the constitutive relation (5) takes the form

$$
\sigma_{x x}=(\lambda+2 \mu) \frac{\partial u}{\partial x}-\gamma \theta
$$

The equation of motion, heat transport equation, and relation between conductive temperature and thermodynamic temperature can be written as

$$
\begin{gathered}
\rho \ddot{u}=(\lambda+2 \mu) \frac{\partial^{2} u}{\partial x^{2}}-\gamma \frac{\partial \tilde{\theta}}{\partial x}, \\
I^{\xi-1}\left(1+\tau_{T} \frac{\partial}{\partial t}+\frac{\tau_{T}^{2}}{2} \frac{\partial^{2}}{\partial t^{2}}\right) \frac{\partial^{2} \tilde{\phi}}{\partial x^{2}} \\
=\left(1+\tau_{q} \frac{\partial}{\partial t}+\frac{\tau_{q}^{2}}{2} \frac{\partial^{2}}{\partial t^{2}}\right)\left(\frac{\dot{\tilde{\theta}}}{\kappa}+\frac{\gamma T_{0}}{K_{0}} \dot{e}\right), \\
\tilde{\phi}-\tilde{\theta}=a \frac{\partial^{2} \tilde{\phi}}{\partial x^{2}} .
\end{gathered}
$$

We now use the following nondimensional variables, to make the above equations nondimensional:

$$
\begin{array}{ll}
x^{\prime}=\frac{c_{0}}{\kappa} x, & u^{\prime}=\frac{c_{0}}{\kappa} u, \quad t^{\prime}=\frac{c_{0}^{2}}{\kappa} t, \\
\tau_{T}^{\prime}=\frac{c_{0}^{2}}{\kappa} \tau_{T}, & \tau_{q}^{\prime}=\frac{c_{0}^{2}}{\kappa} \tau_{q}, \quad \sigma_{x x}^{\prime}=\frac{\sigma_{x x}}{\lambda+2 \mu}, \\
\phi^{\prime}=\frac{\phi}{T_{0}}, & \widetilde{\phi}^{\prime}=\frac{\tilde{\phi}}{T_{0}}, \quad \theta^{\prime}=\frac{\theta}{T_{0}}, \\
\widetilde{\theta}^{\prime}=\frac{\tilde{\theta}}{T_{0}}, & c_{0}^{2}=\frac{\lambda+2 \mu}{\rho} .
\end{array}
$$


Then the corresponding nondimensional equations, after omitting the primes, are

$$
\begin{gathered}
\sigma_{x x}=\frac{\partial u}{\partial x}-\epsilon_{2} \theta \\
\ddot{u}=\frac{\partial^{2} u}{\partial x^{2}}-\epsilon_{2} \frac{\partial \tilde{\theta}}{\partial x} \\
I^{\xi-1}\left(1+\tau_{T} \frac{\partial}{\partial t}+\frac{\tau_{T}^{2}}{2} \frac{\partial^{2}}{\partial t^{2}}\right) \frac{\partial^{2} \tilde{\phi}}{\partial x^{2}} \\
=\left(1+\tau_{q} \frac{\partial}{\partial t}+\frac{\tau_{q}^{2}}{2} \frac{\partial^{2}}{\partial t^{2}}\right)\left(\dot{\tilde{\theta}}+\epsilon_{1} \dot{e}\right), \\
\tilde{\phi}-\tilde{\theta}=\beta \frac{\partial^{2} \tilde{\phi}}{\partial x^{2}}
\end{gathered}
$$

where

$$
\epsilon_{1}=\frac{\gamma \kappa}{K_{0}}, \quad \epsilon_{2}=\frac{\gamma T_{0}}{(\lambda+2 \mu)}, \quad \beta=\frac{a c_{0}^{2}}{\kappa^{2}} .
$$
by

Initial and regularity conditions for the problem are given

$$
\begin{gathered}
u=\theta=\phi=0 \quad \text { at } t=0 \text { for } x \geq 0, \\
\frac{\partial u}{\partial t}=\frac{\partial \theta}{\partial t}=\frac{\partial \phi}{\partial t}=0 \quad \text { at } t=0 \text { for } x \geq 0, \\
u=\theta=\phi=0 \quad \text { as } x \longrightarrow \infty .
\end{gathered}
$$

\section{Method of Solution}

Applying the Laplace transform defined by

$$
\bar{f}(s)=\int_{0}^{\infty} e^{-s t} f(t) d t, \quad \operatorname{Re}(s)>0
$$

to both the sides of (32), we obtain

$$
\begin{gathered}
\bar{\sigma}_{x x}=\bar{e}-\epsilon_{2} \bar{\theta}, \\
\frac{d^{2} \bar{e}}{d x^{2}}=s^{2} \bar{e}+\epsilon_{2} \frac{d^{2} \overline{\widetilde{\theta}}}{d x^{2}}, \\
\left(1+\tau_{T} s+\frac{1}{2} \tau_{T}^{2} s^{2}\right) \frac{d^{2} \overline{\widetilde{\phi}}}{d x^{2}} \\
=s^{\xi}\left(1+\tau_{q} s+\frac{1}{2} \tau_{q}^{2} s^{2}\right)\left(\overline{\widetilde{\theta}}+\epsilon_{1} \bar{e}\right), \\
\overline{\widetilde{\phi}}-\overline{\widetilde{\theta}}=\beta \frac{d^{2} \overline{\widetilde{\phi}}}{d x^{2}} .
\end{gathered}
$$

Eliminating $\overline{\widetilde{\theta}}$ from (37)-(39) we get

$$
\begin{aligned}
& \frac{d^{2} \overline{\tilde{\phi}}}{d x^{2}}=\alpha_{1} \overline{\widetilde{\phi}}+\epsilon_{1} \alpha_{1} \bar{e}, \\
& \frac{d^{2} \bar{e}}{d x^{2}}=\alpha_{2} \overline{\widetilde{\phi}}+\alpha_{3} \bar{e},
\end{aligned}
$$

where

$$
\begin{aligned}
a & =\left(\frac{1+\tau_{q} s+(1 / 2) \tau_{q}^{2} s^{2}}{1+\tau_{T} s+(1 / 2) \tau_{T}^{2} s^{2}}\right) \\
\alpha_{1} & =\frac{a s^{\xi}}{1+a \beta s^{\xi}}, \quad \alpha_{2}=\frac{\alpha_{1} \epsilon_{2}\left(1-\beta \alpha_{1}\right)}{\left[1+\beta \alpha_{1} \epsilon_{1} \epsilon_{2}\right]}, \\
\alpha_{3} & =\frac{s^{2}+\alpha_{1} \epsilon_{1} \epsilon_{2}\left(1-\beta \alpha_{1}\right)}{\left[1+\beta \alpha_{1} \epsilon_{1} \epsilon_{2}\right]} .
\end{aligned}
$$

5.1. State Space Approach. The equations (40) can be written in the form of a vector matrix differential equations [60] as follows:

$$
\frac{d^{2} \bar{V}(x, s)}{d x^{2}}=A(s) \bar{V}(x, s),
$$

where

$$
\bar{V}(x, s)=\left[\begin{array}{c}
\bar{\phi}(x, s) \\
\bar{e}(x, s)
\end{array}\right], \quad A(s)=\left[\begin{array}{cc}
\alpha_{1} & \epsilon_{1} \alpha_{1} \\
\alpha_{2} & \alpha_{3}
\end{array}\right] .
$$

The formal solution of system (42) bounded at infinity can be written as

$$
\bar{V}(x, s)=\exp [-\sqrt{A(s)} x] \bar{V}(0, s),
$$

where

$$
\begin{aligned}
& \bar{V}(0, s)=\left[\begin{array}{l}
\overline{\widetilde{\phi}}(0, s) \\
\bar{e}(0, s)
\end{array}\right]=\left[\begin{array}{l}
\overline{\tilde{\phi}}_{0} \\
\bar{e}_{0}
\end{array}\right], \\
& \phi_{0}=\phi(0, t), \quad e_{0}=e(0, t) .
\end{aligned}
$$

We will use the well-known Cayley-Hamilton theorem to find the form of the matrix

$$
\exp [-\sqrt{A(s)} x]
$$

The characteristic equation for the matrix $A(s)$ can be written as

$$
k^{2}-k\left(\alpha_{1}+\alpha_{3}\right)+\left(\alpha_{1} \alpha_{3}-\epsilon_{1} \alpha_{1} \alpha_{2}\right)=0 .
$$

The roots of this equation, namely, $k_{1}$ and $k_{2}$, satisfy the following relations:

$$
\begin{gathered}
k_{1}+k_{2}=\alpha_{1}+\alpha_{3}, \\
k_{1} k_{2}=\alpha_{1} \alpha_{3}-\epsilon_{1} \alpha_{1} \alpha_{2} .
\end{gathered}
$$

The Taylor series expansion for the matrix exponential in (44) is given by

$$
\exp [-\sqrt{A(s)} x]=\sum_{n=0}^{\infty} \frac{[-\sqrt{A(s)} x]^{n}}{n !}
$$

Using Cayley-Hamilton theorem, we can express $A^{2}$ and higher powers of the matrix $A$ in terms of $I$ and $A$, where $I$ is the unit matrix of the second order. 
Thus the infinite series in (49) can be reduced to the form

$$
\exp [-\sqrt{A(s)} x]=a_{0}(x, s) I+a_{1}(x, s) A(s),
$$

where $a_{0}$ and $a_{1}$ are coefficients depending on $s$ and $x$.

By Cayley-Hamilton theorem, the characteristic roots $k_{1}$ and $k_{2}$ of the matrix $A$ must satisfy (50); thus we have

$$
\begin{aligned}
& \exp \left(-\sqrt{k_{1}} x\right)=a_{0}+a_{1} k_{1}, \\
& \exp \left(-\sqrt{k_{2}} x\right)=a_{0}+a_{1} k_{2} .
\end{aligned}
$$

By solving the above linear system of equations, we get

$$
\begin{aligned}
& a_{0}=\frac{k_{1} e^{-\sqrt{k_{2}} x}-k_{2} e^{-\sqrt{k_{1}} x}}{k_{1}-k_{2}}, \\
& a_{1}=\frac{e^{-\sqrt{k_{1}} x}-e^{-\sqrt{k_{2}} x}}{k_{1}-k_{2}} .
\end{aligned}
$$

Hence from (50) we get

$$
\exp [-\sqrt{A(s)} x]=L_{i j}(x, s), \quad i, j=1,2,
$$

where

$$
\begin{aligned}
& L_{11}=\frac{e^{-\sqrt{k_{2}} x}\left(k_{1}-\alpha_{1}\right)-e^{-\sqrt{k_{1}} x}\left(k_{2}-\alpha_{1}\right)}{k_{1}-k_{2}}, \\
& L_{12}=\frac{\epsilon_{1} \alpha_{1}\left(e^{-\sqrt{k_{1}} x}-e^{-\sqrt{k_{2}} x}\right)}{k_{1}-k_{2}}, \\
& L_{21}=\frac{\alpha_{2}\left(e^{-\sqrt{k_{1}} x}-e^{-\sqrt{k_{2}} x}\right)}{k_{1}-k_{2}}, \\
& L_{22}=\frac{e^{-\sqrt{k_{1}} x}\left(\alpha_{3}-k_{2}\right)-e^{-\sqrt{k_{2}} x}\left(\alpha_{3}-k_{1}\right)}{k_{1}-k_{2}} .
\end{aligned}
$$

Using (53) we can write the solution in (44) in the following form:

$$
\bar{V}(x, s)=L_{i j}(x, s) \bar{V}(0, s) .
$$

Hence the solution for $\overline{\widetilde{\phi}}(x, s)$ and $\bar{e}(x, s)$ can be obtained from (55) as follows:

$$
\begin{array}{r}
\overline{\widetilde{\phi}}(x, s)=\frac{1}{k_{1}-k_{2}}\left[e^{-\sqrt{k_{1}} x}\left\{\epsilon_{1} \alpha_{1} \bar{e}_{0}-\left(k_{2}-\alpha_{1}\right) \overline{\widetilde{\phi}}_{0}\right\}\right. \\
\left.-e^{-\sqrt{k_{2}} x}\left\{\epsilon_{1} \alpha_{1} \bar{e}_{0}-\left(k_{1}-\alpha_{1}\right) \overline{\widetilde{\phi}}_{0}\right\}\right], \\
\bar{e}(x, s)=\frac{1}{k_{1}-k_{2}}\left[e^{-\sqrt{k_{1}} x}\left\{\alpha_{2} \overline{\widetilde{\phi}}_{0}-\left(k_{2}-\alpha_{3}\right) \bar{e}_{0}\right\}\right. \\
\left.-e^{-\sqrt{k_{2}} x}\left\{\alpha_{2} \overline{\widetilde{\phi}}_{0}-\left(k_{1}-\alpha_{3}\right) \bar{e}_{0}\right\}\right] .
\end{array}
$$

Using (56), the solution for $\overline{\widetilde{\theta}}$ can be obtained from (39) as follows:

$$
\begin{aligned}
\overline{\widetilde{\theta}}=\frac{1}{k_{1}-k_{2}}\left[e^{-\sqrt{k_{1}} x}\left\{\epsilon_{1} \alpha_{1} \bar{e}_{0}-\left(k_{2}-\alpha_{1}\right) \overline{\widetilde{\phi}}_{0}\right\}\left(1-\beta k_{1}\right)\right. \\
\left.-e^{-\sqrt{k_{2}} x}\left\{\epsilon_{1} \alpha_{1} \bar{e}_{0}-\left(k_{1}-\alpha_{1}\right) \overline{\widetilde{\phi}}_{0}\right\}\left(1-\beta k_{2}\right)\right] .
\end{aligned}
$$

5.2. Application to Thermal Shock Problem. We will consider the bounding plane of the medium at $x=0$ subjected to thermal shock in the following nondimensional form:

$$
\phi(0, t)=\phi_{1} H(t)
$$

where $\phi_{1}$ is constant. Now applying the Laplace transform to (59) we get

$$
\bar{\phi}(0, s)=\frac{\phi_{1}}{s} .
$$

Using (9), (11), and (60) we get

$$
\overline{\widetilde{\phi}}(0, s)=\frac{l}{s}
$$

where $l=\phi_{1}+\left(K_{1} / 2 s\right) \phi_{1}^{2}$.

5.3. The Mechanical Boundary Condition. The mechanical boundary condition is taken in the form

$$
e(0, t)=0
$$

This implies

$$
\bar{e}(0, s)=\bar{e}_{0}=0
$$

Applying the boundary conditions (61) and (63) to (56)-(58) we get

$$
\begin{gathered}
\overline{\widetilde{\phi}}=\frac{l}{s\left(k_{1}-k_{2}\right)}\left[\left(\alpha_{1}-k_{2}\right) e^{-\sqrt{k_{1}} x}-\left(\alpha_{1}-k_{1}\right) e^{-\sqrt{k_{2}} x}\right], \\
\bar{e}=\frac{l \alpha_{2}}{s\left(k_{1}-k_{2}\right)}\left[e^{-\sqrt{k_{1}} x}-e^{-\sqrt{k_{2}} x}\right], \\
\overline{\tilde{\theta}}=\frac{l}{s\left(k_{1}-k_{2}\right)}\left[\left(\alpha_{1}-k_{2}\right)\left(1-\beta k_{1}\right) e^{-\sqrt{k_{1}} x}\right. \\
\left.\quad-\left(\alpha_{1}-k_{1}\right)\left(1-\beta k_{2}\right) e^{-\sqrt{k_{2}} x}\right] .
\end{gathered}
$$

Displacement component $\bar{u}$ can be obtained from (28) using (65) in the following form:

$$
\bar{u}=\frac{-l \alpha_{2}}{s\left(k_{1}-k_{2}\right)}\left[\frac{e^{-\sqrt{k_{1}} x}}{\sqrt{k_{1}}}-\frac{e^{-\sqrt{k_{2}} x}}{\sqrt{k_{2}}}\right] .
$$


Using (9), (11), and (64) the solution for $\bar{\phi}$ can be obtained as follows:

$$
\begin{gathered}
\bar{\phi}=\frac{1}{K_{1}}\left[\left(1+\frac{2 K_{1} l}{s\left(k_{1}-k_{2}\right)}\right.\right. \\
\times\left[\left(\alpha_{1}-k_{2}\right) e^{-\sqrt{k_{1}} x}\right. \\
\left.\left.\left.-\left(\alpha_{1}-k_{1}\right) e^{-\sqrt{k_{2}} x}\right]\right)^{1 / 2}-1\right], \quad K_{1}<0 \\
=\frac{l}{s\left(k_{1}-k_{2}\right)}\left[\left(\alpha_{1}-k_{2}\right) e^{-\sqrt{k_{1}} x}\right. \\
\left.-\left(\alpha_{1}-k_{1}\right) e^{-\sqrt{k_{2}} x}\right], \quad K_{1}=0 .
\end{gathered}
$$

Again using (9), (12), and (66) the solution for $\bar{\theta}$ can be obtained in the following form:

$$
\begin{aligned}
\bar{\theta}= & \frac{l}{s\left(k_{1}-k_{2}\right)}\left[\left(\alpha_{1}-k_{2}\right)\left(1-\beta k_{1}\right) e^{-\sqrt{k_{1}} x}\right. \\
& \left.\quad-\left(\alpha_{1}-k_{1}\right)\left(1-\beta k_{2}\right) e^{-\sqrt{k_{2}} x}\right] \\
= & \widetilde{\theta}_{0}(\text { say }), \quad K_{1}=0 \\
= & \frac{1}{K_{1}}\left[\sqrt{1+2 K_{1} \widetilde{\theta}_{0}}-1\right], \quad K_{1}<0 .
\end{aligned}
$$

The solution for $\bar{\sigma}_{x x}$ can be obtained from (36) using (65) and (69) in the following form:

$$
\begin{aligned}
& \bar{\sigma}_{x x}= \frac{l \alpha_{2}}{s\left(k_{1}-k_{2}\right)}\left[e^{-\sqrt{k_{1}} x}-e^{-\sqrt{k_{2}} x}\right] \\
&-\frac{\epsilon_{2}}{K_{1}}\left[\sqrt{1+2 K_{1} \widetilde{\theta}_{0}}-1\right], \quad K_{1}<0 ; \\
&= \frac{l \alpha_{2}}{s\left(k_{1}-k_{2}\right)}\left[e^{-\sqrt{k_{1}} x}-e^{-\sqrt{k_{2}} x}\right]-\epsilon_{2} \widetilde{\theta}_{0}, \\
& K_{1}=0 .
\end{aligned}
$$

This completes the solution of the thermal shock problem in Laplace transform domain.

\section{Numerical Inversion of Laplace Transform}

It is difficult to find the analytical inverse of Laplace transform of the complicated solutions for the displacement, thermodynamic temperature, conductive temperature, stress, and strain in Laplace transform domain. So we have to resort to numerical computations. We now outline the numerical procedure to solve the problem. Let $\bar{f}(x, s)$ be the Laplace transform of a function $f(x, t)$.

Then the inversion formula for Laplace transform can be written as

$$
f(x, t)=\frac{1}{2 \pi i} \int_{d-i \infty}^{d+i \infty} e^{s t} \bar{f}(x, s) d s,
$$

where $d$ is an arbitrary real number greater than real parts of all the singularities of $\bar{f}(x, s)$.

Taking $s=d+i w$, the preceding integral takes the form

$$
f(x, t)=\frac{e^{d t}}{2 \pi} \int_{-\infty}^{\infty} e^{i t w} f(x, d+i w) d w
$$

Expanding the function $h(x, t)=e^{-d t} f(x, t)$ in a Fourier series in the interval $[0,2 T]$ we obtain the approximate formula [32]

$$
f(x, t)=f_{\infty}(x, t)+E_{D}
$$

where

$$
\begin{gathered}
f_{\infty}(x, t)=\frac{1}{2} c_{0}+\sum_{k=1}^{\infty} c_{k} \quad \text { for } 0 \leq t \leq 2 T, \\
c_{k}=\frac{e^{d t}}{T}\left[e^{i k \pi t / T} \bar{f}\left(x, d+\frac{i k \pi t}{T}\right)\right] .
\end{gathered}
$$

The discretization error $E_{D}$ can be made arbitrary small by choosing $d$ large enough [32]. Since the infinite series in (74) can be summed up to a finite number $N$ of terms, the approximate value of $f(x, t)$ becomes

$$
f_{N}(x, t)=\frac{1}{2} c_{0}+\sum_{k=1}^{N} c_{k} \quad \text { for } 0 \leq t \leq 2 T .
$$

Using the preceding formula to evaluate $f(x, t)$ we introduce a truncation error $E_{T}$ that must be added to the discretization error to produce total approximation error.

Two methods are used to reduce the total error. First the "Korrektur" method is used to reduce the discretization error. Next the $\varepsilon$-algorithm is used to accelerate convergence [32].

The Korrektur method uses the following formula to evaluate the function $f(x, t)$ :

$$
f(x, t)=f_{\infty}(x, t)-e^{-2 d T} f_{\infty}(x, 2 T+t)+E_{D^{\prime}}^{\prime}
$$

where the discretization error $\left|E_{D}^{\prime}\right| \ll\left|E_{D}\right|$. Thus, the approximate value of $f(x, t)$ becomes

$$
f_{N K}(x, t)=f_{N}(x, t)-e^{-2 d T} f_{N^{\prime}}(x, 2 T+t),
$$

where $N^{\prime}$ is an integer such that $N^{\prime}<N$.

We will now describe the $\varepsilon$-algorithm that is used to accelerate the convergence of the series in (75). Let $N=$ $2 q+1$, where $q$ is a natural number, and let $s_{m}=\sum_{k=1}^{m} c_{k}$ be the sequence of partial sum of series in (75).

We define the $\varepsilon$-sequence by

$$
\begin{gathered}
\varepsilon_{0, m}=0, \quad \varepsilon_{1, m}=s_{m}, \\
\varepsilon_{p+1, m}=\varepsilon_{p-1, m+1}+\frac{1}{\varepsilon_{p, m+1}-\varepsilon_{p, m}}, \quad p=1,2,3, \ldots .
\end{gathered}
$$

It can be shown that [32] the sequence

$$
\varepsilon_{1,1}, \varepsilon_{3,1}, \varepsilon_{5,1}, \ldots, \varepsilon_{N, 1}
$$




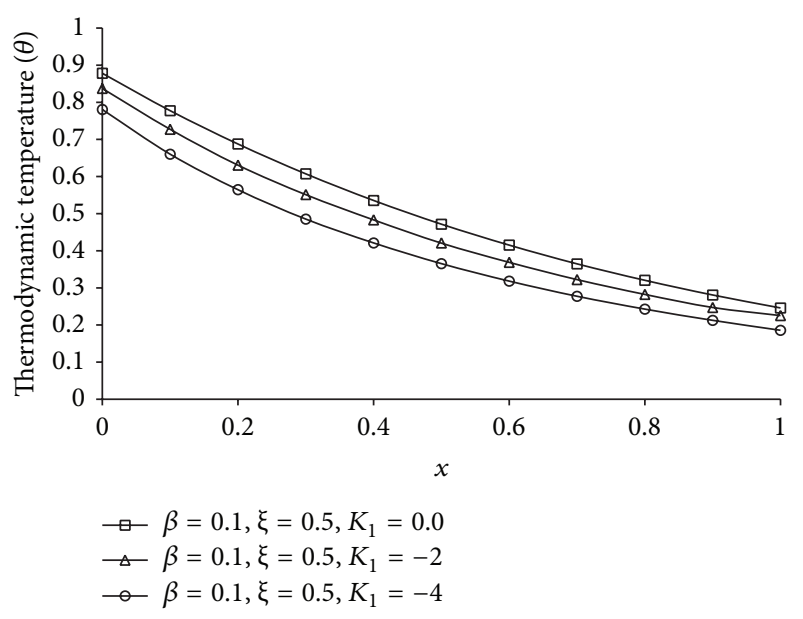

FIgURE 1: The thermodynamic temperature $(\theta)$ for different $K_{1}$ at $t=0.2$.

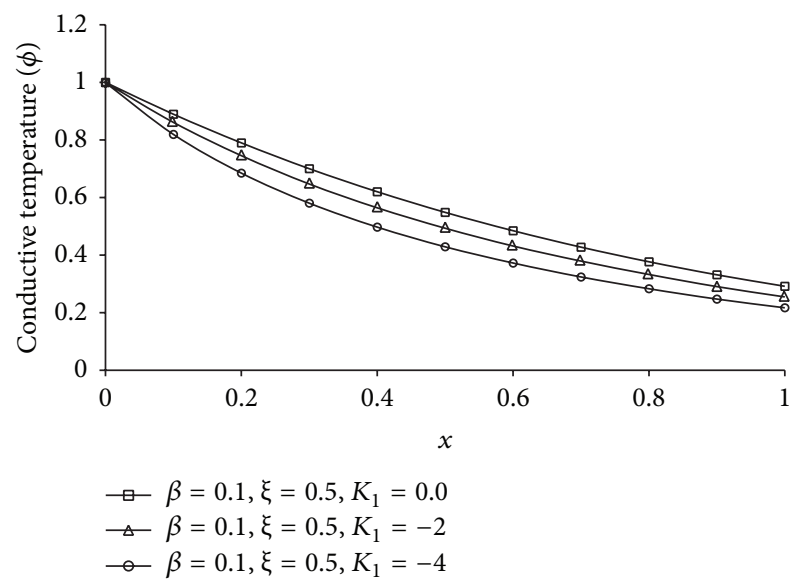

Figure 2: The conductive temperature $(\phi)$ for different $K_{1}$ at $t=0.2$.

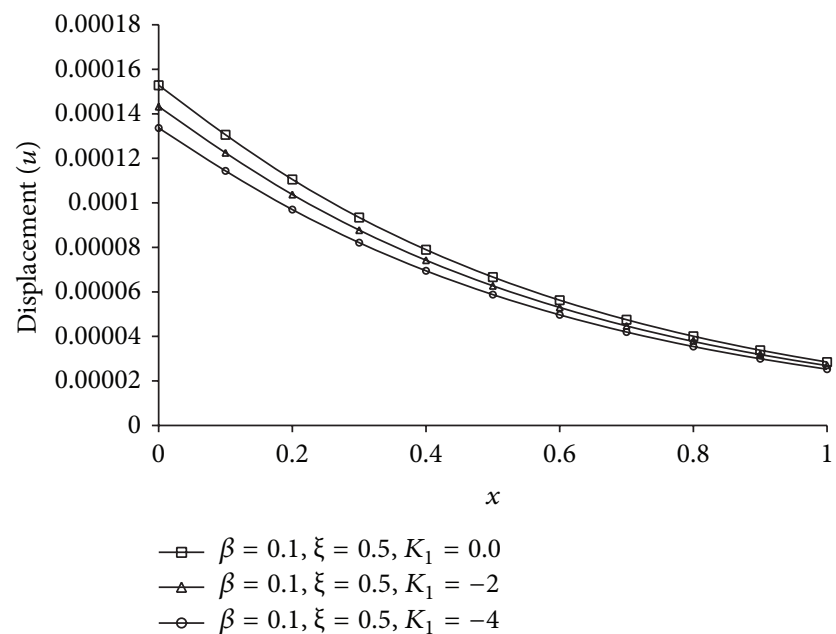

FIGURE 3: The displacement $(u)$ for different $K_{1}$ at $t=0.2$.

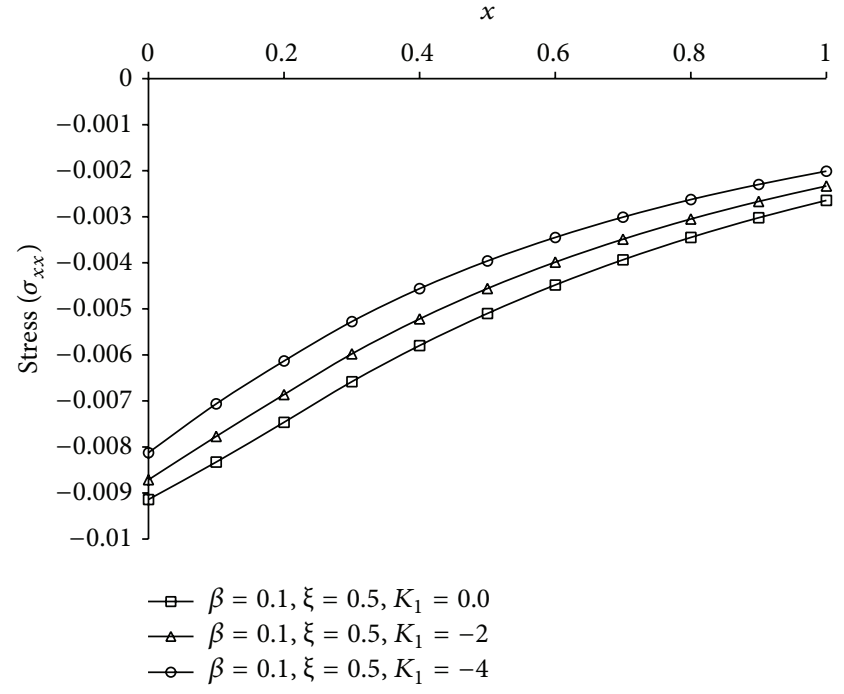

Figure 4: The stress $\left(\sigma_{x x}\right)$ for different $K_{1}$ at $t=0.2$.

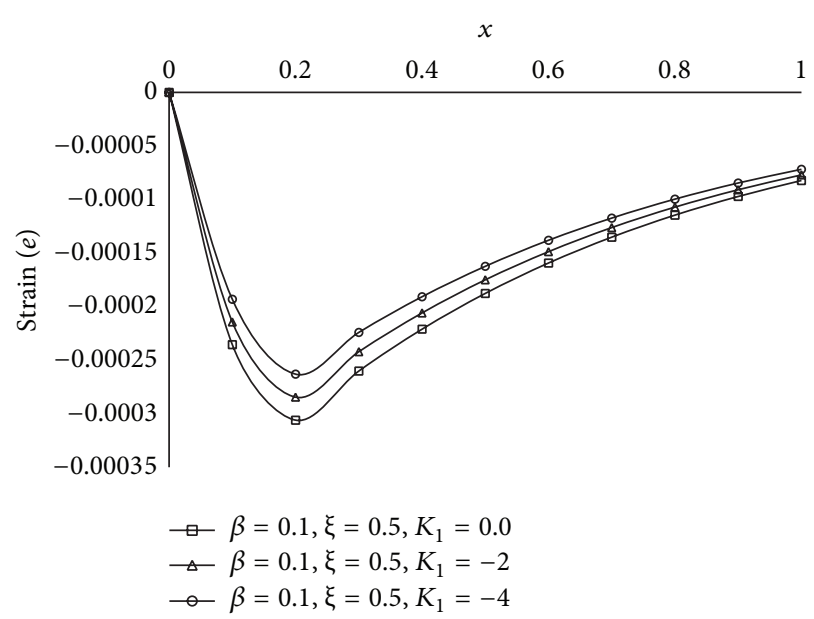

Figure 5: The strain (e) for different $K_{1}$ at $t=0.2$.

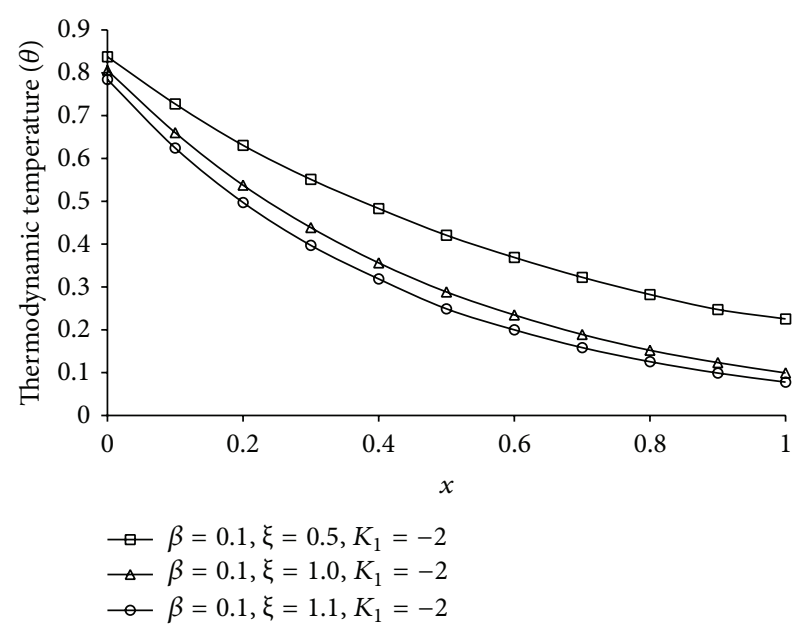

FIgURE 6: The thermodynamic temperature $(\theta)$ for different $\xi$ at $t=$ 0.2 . 


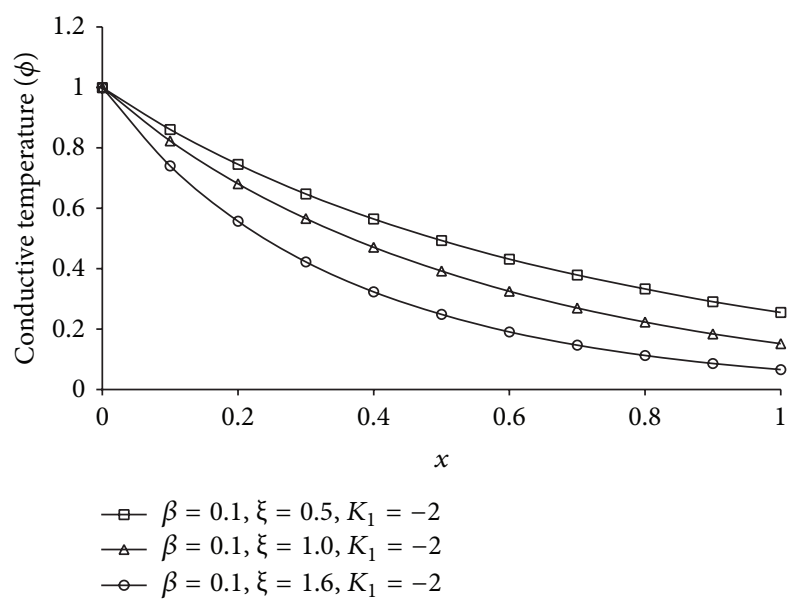

FIgURE 7: The conductive temperature $(\phi)$ for different $\xi$ at $t=0.2$.

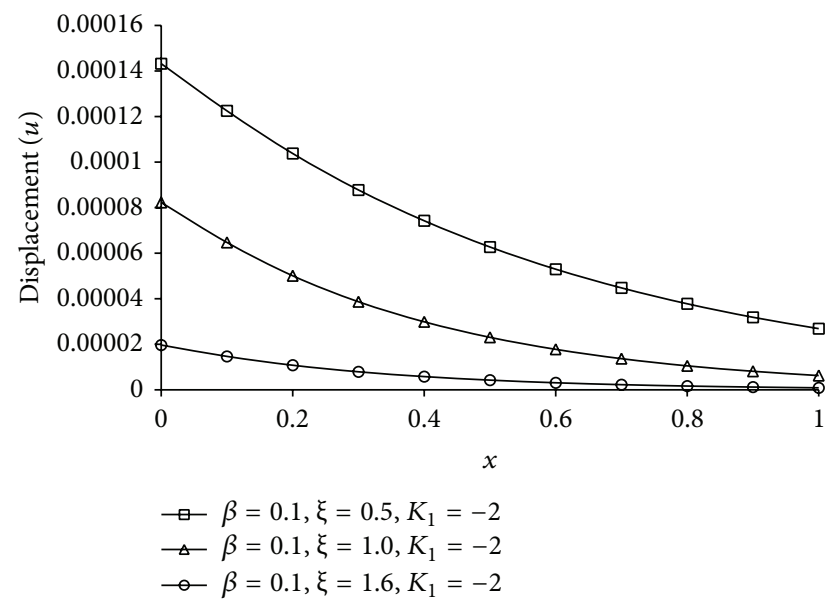

FIGURE 8: The displacement $(u)$ for different $\xi$ at $t=0.2$.

converges to $f(x, t)+E_{D}-\left(c_{0} / 2\right)$ faster than the sequence of partial sums $s_{m}, m=1,2,3, \ldots$.

The actual procedure used to invert the Laplace transform consists of using (77) together with the $\varepsilon$-algorithm. The values of $d$ and $T$ are chosen according to the criterion outlined in [32].

\section{Numerical Results and Discussion}

To get the solution for strain $(e)$, thermal displacement component $(u)$, conductive temperature $(\phi)$, thermodynamic temperature $(\theta)$, and thermal stress $\left(\sigma_{x x}\right)$ in the space time domain we have applied Laplace inversion formula to (65), (67), (68), (69), and (70), respectively, which have been done numerically using a method based on Fourier series expansion technique [32]. The numerical code has been prepared using Fortran 77 programming language. For computational

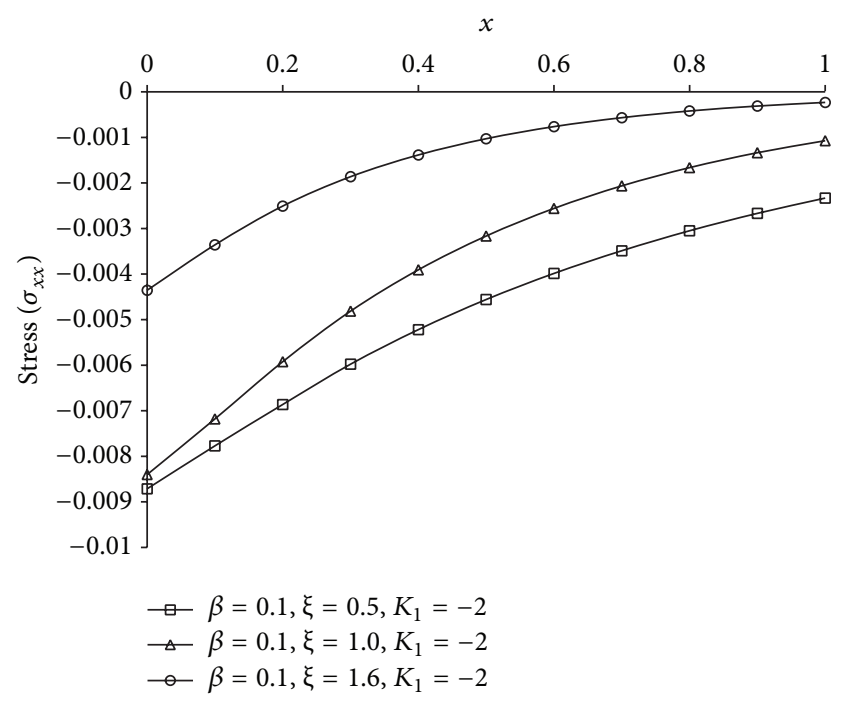

Figure 9: The stress $\left(\sigma_{x x}\right)$ for different $\xi$ at $t=0.2$.

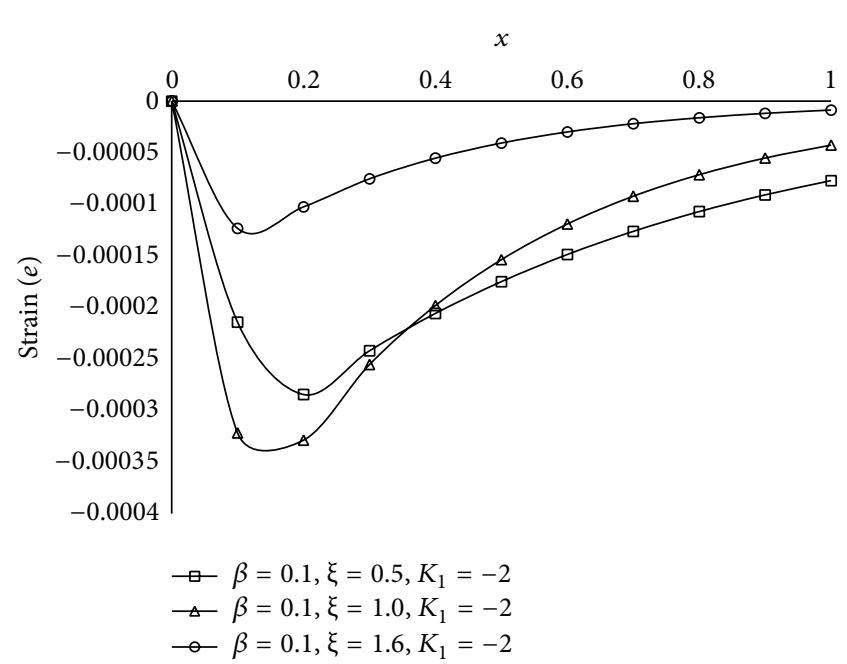

Figure 10: The strain $(e)$ for different $\xi$ at $t=0.2$.

purpose copper material has been taken into consideration. The values of the material constants are taken as follows [61]:

$$
\begin{aligned}
\lambda & =7.76 \times 10^{10} \mathrm{Nm}^{-2}, \\
\mu & =3.86 \times 10^{10} \mathrm{Nm}^{-2}, \\
\rho & =8954 \mathrm{Kg} \mathrm{m}^{-3}, \\
K_{0} & =386 \mathrm{Wm}^{-1} \mathrm{~K}^{-1}, \\
c_{E} & =383.1 \mathrm{JKg}^{-1} \mathrm{~K}^{-1}, \quad T_{0}=293 \mathrm{~K}, \\
\alpha_{t} & =1.78 \times 10^{-5} \mathrm{~K}^{-1}, \quad \varepsilon_{1}=1.618, \\
\varepsilon_{2} & =0.01041, \quad \beta=0.1, \\
\tau_{q} & =0.02, \quad \tau_{T}=0.015 .
\end{aligned}
$$

Also we take time $t=0.2, \phi_{1}=1$ for computational purpose. 


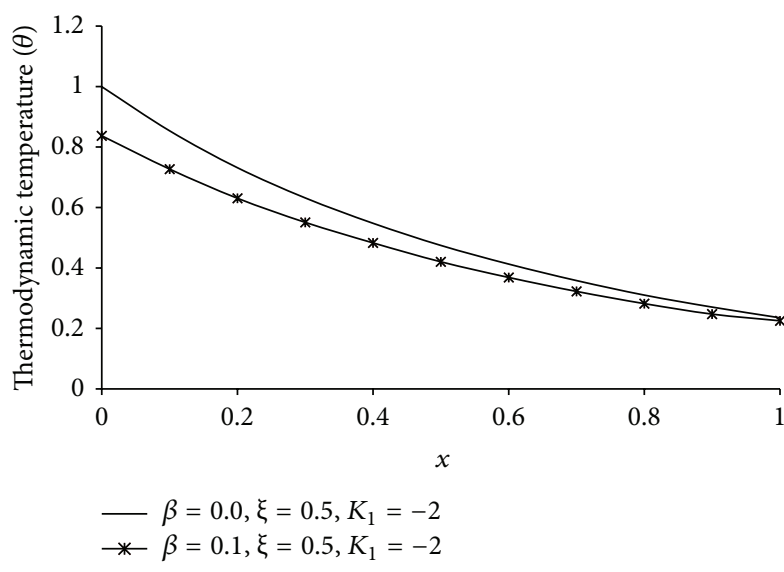

FIgURE 11: The comparison of thermodynamic temperature $(\theta)$ between 1TT and 2TT at $t=0.2$.

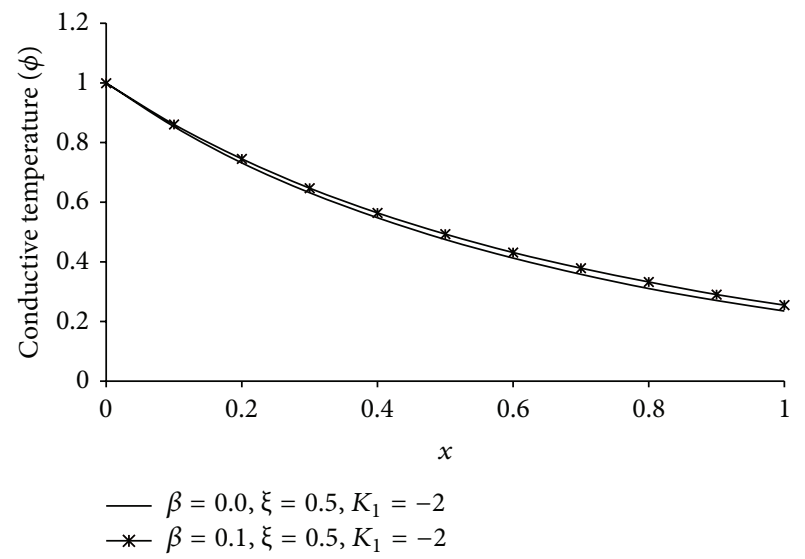

FIGURE 12: The comparison of conductive temperature $(\phi)$ between 1TT and 2TT at $t=0.2$.

Figures 1, 2, 3, 4, 5, 6, 7, 8, 9, 10, 11, 12, 13, 14, and 15 are drawn to represent the variation of said thermophysical quantities versus the space variable $x$ for different $K_{1}(=0,-2,-4), \xi(=0.5,1.0,1.6)$, and $\beta(=0.0,0.1)$. Here $\xi=0.5,1.0$, and 1.6 corresponds to week conductivity, normal conductivity, and super conductivity, respectively; $\beta=0.0$ and 0.1 corresponds to one-temperature and twotemperature theory, respectively.

Figures 1-5 show the effect of $K_{1}$ on the said five thermophysical quantities for two-temperature theory $(\beta=$ 0.1 ) and fractional order parameter $\xi=0.5$. From these figures it is clear that magnitude of all the quantities, that is, thermodynamic temperature $\theta$, conductive temperature $\phi$, displacement $u$, stress component $\sigma$, and strain component $e$, is greater for smaller magnitude of $K_{1}$.

Figures 6-10 show the effect of $\xi$ for two-temperature $(\beta=$ 0.1 ) theory and $K_{1}=-2$ on those five quantities. Figures 6-9 show that the magnitude of thermodynamic temperature $\theta$, conductive temperature $\phi$, displacement component $u$, and stress component $\sigma$ has greater value for smaller magnitude of $\xi$. But from Figure 10 it is observed that in the region $0.0 \leq$

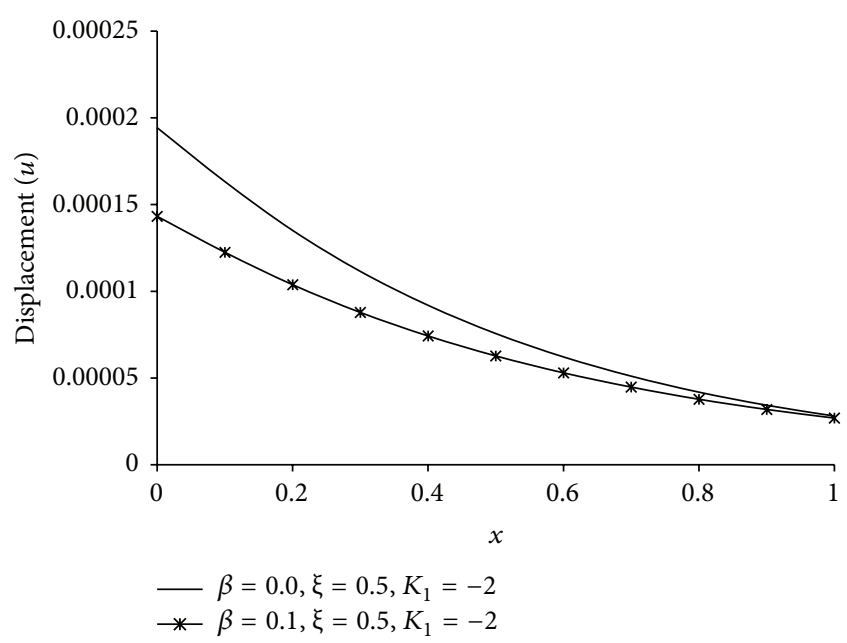

FIGURE 13: The comparison of displacement $(u)$ between 1TT and $2 \mathrm{TT}$ at $t=0.2$.

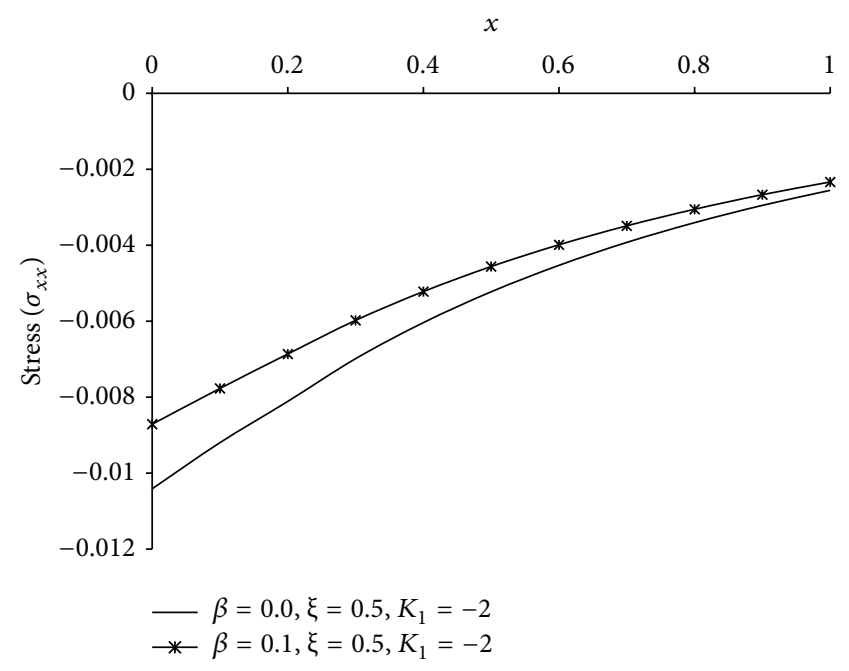

FIGURE 14: The comparison of stress $\left(\sigma_{x x}\right)$ between 1TT and 2TT at $t=0.2$.

$x<0.3$ (approximate) strain component has larger value for $\xi=1.0$ than for $\xi=0.5$, which is again larger than for $\xi=1.6$.

Figures 11-15 are drawn to compare between the results of one-temperature $(\beta=0.0)$ theory and two-temperature ( $\beta=0.1$ ) theory for $\xi=0.5$ and $K_{1}=-2$ for five different thermophysical quantities. Figures 11, 13, 14, and 15 show that the magnitude of $\theta, u, \sigma, e$ is greater for one-temperature $(\beta=0.0)$ case than two-temperature $(\beta=0.1)$ case. The only exception is $\phi$ here.

It is observed that at the boundary plane $x=0, \phi=1$ (Figures 2, 7, and 12), and $e=0$ (Figures 5, 10, and 15), which satisfies our theoretical boundary condition. It ensures the correctness of the numerical code used. In Figures 6-10 the results for $\xi=1$ agree with the corresponding results of Mondal et al. [24].

Figures 16 and 17 represent the variation of the thermodynamic temperature $\theta$ and conductive temperature $\phi$ against 


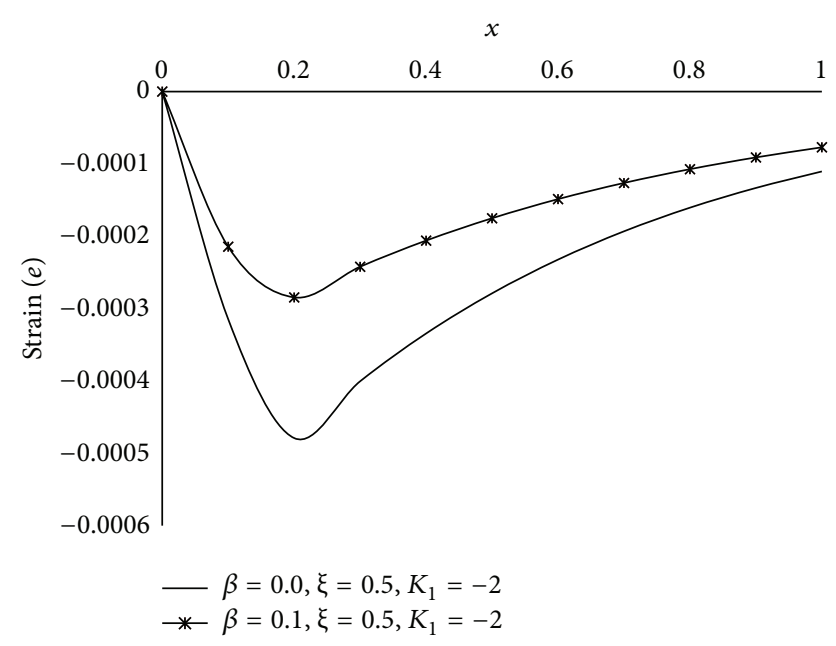

FIGURE 15: The comparison of radial strain $(e)$ between 1TT and 2TT at $t=0.2$.

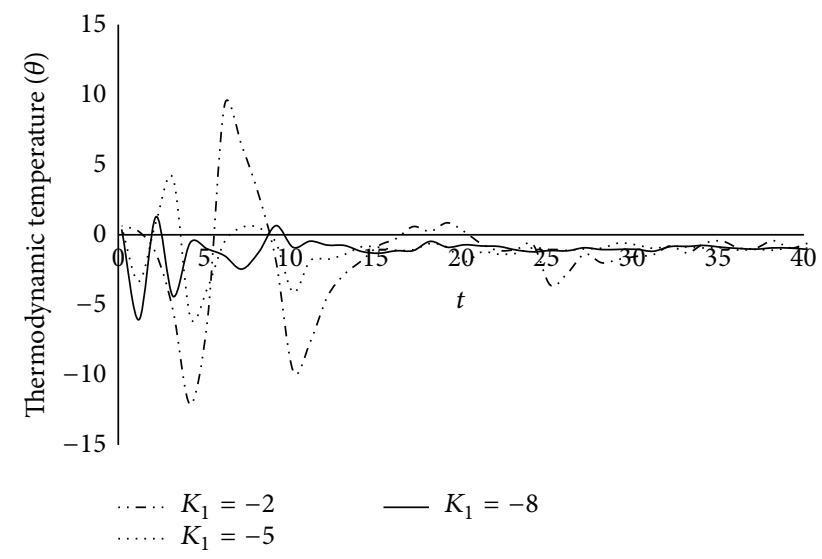

FIGURE 16: The variation of thermodynamic temperature $(\theta)$ with time $(t)$ at $x=0.2$ for $\beta=0.1 ; \xi=0.5$.

time $t$ for different value of $K_{1}$, namely, $K_{1}=-2,-5,-8$, when $\beta=0.1, \xi=0.5$, and $x=0.2$. It is observed from the figures that the magnitudes of $\theta$ and $\phi$ are oscillatory in nature and the magnitude of peek of oscillation decreases with time.

\section{Conclusion}

State space approach has been applied to solve a generalized thermoelastic problem of an isotropic, half-space with variable thermal conductivity. The boundary $(x=0)$ of the half-space is subjected to thermal and mechanical loads. Variation of thermal conductivity has been taken as linear function of temperature. The problem has been studied using the two-temperataure dual-phase-lag model of generalized thermoelasticity in consideration of fractional order heat conduction equation.

(1) The phenomenon of finite speeds of propagation is observed in all depicted figures. This is expected since the thermal wave travels with finite speed.

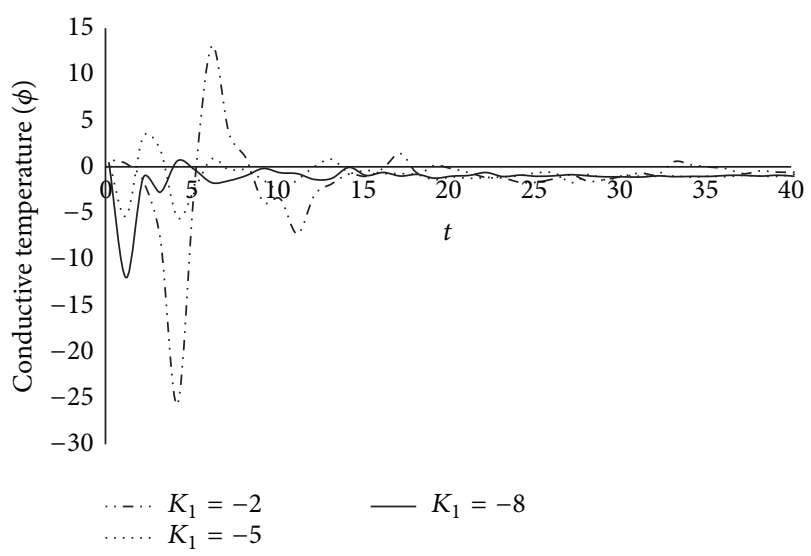

FIGURE 17: The variation of conductive temperature $(\phi)$ with time $(t)$ at $x=0.2$ for $\beta=0.1 ; \xi=0.5$.

(2) The effects of the fractional parameter on all the studied fields are very significant.

(3) The value of $K_{1}$ has an essential role in changing the value of the distributions.

(4) Significant differences in the physical quantities are observed between the one-temperature theory and the two-temperature theory. The two-temperature theory is more realistic than the one-temperature theory in the case of generalized thermoelasticity.

(5) Initially (at $t=0$ ) the conductive temperature has the value of 1 and strain has the value of 0 . These results agree with the boundary conditions.

\section{Nomenclature}

$\begin{array}{ll}\lambda, \mu: & \text { Lamé's constant } \\ \rho: & \text { Density } \\ c_{E}: & \text { Specific heat at constant strain } \\ t: & \text { Time } \\ \xi: & \text { Parameter of the Riemann-Liouville } \\ & \text { fractional integral } \\ \phi: & \text { Conductive temperature } \\ \theta: & \text { Thermodynamic temperature } \\ \alpha_{t}: & \text { Coefficient of linear thermal } \\ \sigma_{i j}: & \text { expansion } \\ e_{i j}: & \text { Components of stress tensor } \\ u_{i}: & \text { Components of strain tensor } \\ K: & \text { Components of displacement vector } \\ \tau_{0}: & \text { Thermal conductivity } \\ c_{0}=\sqrt{(\lambda+2 \mu) / \rho:} & \text { Relaxation time } \\ \kappa=K / \rho c_{E}: & \text { Tongitudinal wave speed } \\ a: & \text { Thermal diffusivity } \\ \beta=a c_{0}^{2} / \kappa^{2}: & \text { Dimensionless two-temperature } \\ \epsilon_{2}=\gamma T_{0} /(\lambda+2 \mu): & \text { parameter } \\ \vec{q}: & \text { Constant } \\ \nu=\lambda / 2(\lambda+\mu): & \text { Heat flux vector } \\ & \text { Poisson's ratio. }\end{array}$




\section{Conflict of Interests}

The authors declare that there is no conflict of interests regarding the publication of this paper.

\section{Acknowledgments}

The authors are grateful to Professor S. C. Bose of the Department of Applied Mathematics, University of Calcutta, for his valuable suggestions and guidance in preparation of the paper. The authors also express their sincere thanks to the reviewer for providing valuable suggestions to improve the paper.

\section{References}

[1] H. W. Lord and Y. A. Shulman, "A generalized dynamical theory of thermoelasticity," Journal of the Mechanics and Physics of Solids, vol. 15, no. 5, pp. 299-309, 1967.

[2] A. E. Green and K. A. Lindsay, "Thermoelasticity," Journal of Elasticity, vol. 2, no. 1, pp. 1-7, 1972.

[3] A. E. Green and P. M. Naghdi, "A re-examination of the basic postulates of thermomechanics," Proceedings of Royal Society of London, vol. 432, no. 1885, pp. 171-194, 1991.

[4] A. E. Green and P. M. Naghdi, "On undamped heat waves in an elastic solid," Journal of Thermal Stresses, vol. 15, no. 2, pp. 253-264, 1992.

[5] A. E. Green and P. M. Naghdi, "Thermoelasticity without energy dissipation," Journal of Elasticity: The Physical and Mathematical Science of Solids, vol. 31, no. 3, pp. 189-208, 1993.

[6] D. Y. Tzou, "Unified field approach for heat conduction from macro- to micro-scales," Journal of Heat Transfer, vol. 117, no. 1, pp. 8-16, 1995.

[7] S. K. Roychoudhuri, "One-dimensional thermoelastic waves in elastic half-space with dual phase-lag effects," Journal of Mechanics of Materials and Structures, vol. 2, no. 3, pp. 489-503, 2007.

[8] R. Quintanilla, "Exponential stability in the dual-phase-lag heat conduction theory," Journal of Non-Equilibrium Thermodynamics, vol. 27, no. 3, pp. 217-227, 2002.

[9] R. Quintanilla, "A condition on the delay parameters in the one-dimensional dual-phase-lag thermoelastic theory," Journal of Thermal Stresses, vol. 26, no. 7, pp. 713-721, 2003.

[10] L. Wang, M. Xu, and X. Zhou, "Well-posedness and solution structure of dual-phase-lagging heat conduction," International Journal of Heat and Mass Transfer, vol. 44, no. 9, pp. 1659-1669, 2001.

[11] L. Wang and M. Xu, "Well-posedness of dual-phase-lagging heat conduction equation: higher dimensions," International Journal of Heat and Mass Transfer, vol. 45, no. 4, pp. 1165-1171, 2002.

[12] L. Wang and X. Mingtian, "Thermal oscillation and resonance in dual-phase-lagging heat conduction," International Journal of Heat and Mass Transfer, vol. 45, no. 5, pp. 1055-1061, 2002.

[13] P. Ailawalia and S. Budhiraja, "Dynamic problem in thermoelastic solid using dual-phase-lag model with internal heat source," Journal of Mathematical Sciences and Applications, vol. 2, no. 1, pp. 10-16, 2014.

[14] M. E. Gurtin and W. O. Williams, "On the Clausius-Duhem inequality," Zeitschrift für angewandte Mathematik und Physik ZAMP, vol. 17, no. 5, pp. 626-633, 1966.
[15] M. E. Gurtin and W. O. Williams, "An axiomatic foundation for continuum thermodynamics," Archive for Rational Mechanics and Analysis, vol. 26, no. 2, pp. 83-117, 1967.

[16] P. J. Chen and M. E. Gurtin, "On a theory of heat conduction involving two temperatures," Zeitschrift für Angewandte Mathematik und Physik, vol. 19, no. 4, pp. 614-627, 1968.

[17] P. J. Chen, M. E. Gurtin, and W. O. Williams, "A note on non simple heat conduction," Zeitschrift für angewandte Mathematik und Physik, vol. 19, no. 6, pp. 969-970, 1968.

[18] P. J. Chen, M. E. Gurtin, and W. O. Williams, "On the thermodynamics of non-simple elastic materials with two temperatures," Zeitschrift für angewandte Mathematik und Physik, vol. 20, no. 1, pp. 107-112, 1969.

[19] W. E. Warren and P. J. Chen, "Wave propagation in the two temperature theory of thermoelasticity," Acta Mechanica, vol. 16, no. 1-2, pp. 21-33, 1973.

[20] D. Lesan, "On the thermodynamics of non-simple elastic materials with two temperatures," Journal of Applied Mathematics and Physics, vol. 21, pp. 583-591, 1970.

[21] P. Puri and P. M. Jordan, "On the propagation of harmonic plane waves under the two-temperature theory," International Journal of Engineering Science, vol. 44, no. 17, pp. 1113-1126, 2006.

[22] R. Quintanilla, "On existence, structural stability, convergence and spatial behavior in thermoelasticity with two temperatures," Acta Mechanica, vol. 168, no. 1-2, pp. 61-73, 2004.

[23] S. Mondal, S. H. Mallik, and M. Kanoria, "Two temperature Green Naghdi models with variable thermal conductivity," Review Bulletin of Calcutta Mathematical Society, vol. 20, no. 2, pp. 121-130, 2012.

[24] S. Mondal, S. H. Mallik, and M. Kanoria, "Two temperature dual-phase-lag model with variable thermal conductivity," Indian Journal of Theoretical Physics, vol. 61, no. 4, pp. 247-262, 2013.

[25] P. Pal, A. Kar, and M. Kanoria, "Effect of three-phase-lag on two temperature Generalized thermoelasticity for homogeneous isotropic half space," International Journal of Mathematical Science and Engineerig Applications, vol. 8, no. 1, pp. 81-104, 2014.

[26] M. Islam, A. Kar, and M. Kanoria, "Two-temperature generalized thermoelasticity in a fiber-reinforced hollow cylinder under thermal shock," International Journal for Computational Methods in Engineering Science and Mechanics, vol. 14, no. 5, pp. 367-390, 2013.

[27] P. Das and M. Kanoria, "Two-Temperature Magneto-thermoelastic response in a perfectly conducting medium based on GN-III model," International Journal of Pure and Applied Mathematics, vol. 81, no. 4, pp. 199-229, 2012.

[28] S. Banik and M. Kanoria, "Two-temperature generalized thermoelastic interactions in an infinite body with a spherical cavity," International Journal of Thermophysics, vol. 32, no. 6, pp. 1247-1270, 2011.

[29] R. Kumar, R. Prasad, and S. Mukhopadhyay, "Some theorems on two-temperature generalized thermoelasticity," Archive of Applied Mechanics, vol. 81, no. 8, pp. 1031-1040, 2011.

[30] R. Kumar, R. Prasad, and S. Mukhopadhyay, "Variational and reciprocal principles in two-temperature generalized thermoelasticity," Journal of Thermal Stresses, vol. 33, no. 3, pp. 161-171, 2010.

[31] P. Ailawalia, S. Budhiraja, and A. Singla, "Dynamic problem in Green-Naghdi (type III) thermoelastic half-space with two temperature," Mechanics of Advanced Materials and Structures, vol. 21, no. 7, pp. 544-552, 2014. 
[32] G. Honig and U. Hirdes, "A method for the numerical inversion of Laplace transforms," Journal of Computational and Applied Mathematics, vol. 10, no. 1, pp. 113-132, 1984.

[33] M. Caputo, "Linear models of dissipation whose Q is almost frequently independent II," Geophys Journal of Royal Astronomical Society, vol. 13, pp. 529-539, 1967.

[34] A. Carpinteri and F. Mainardi, Eds., Fractals and Fractional Calculus in Continuum Mechanics, Springer, New York, NY, USA, 1997.

[35] I. Podlubny, Fractional Differential Equations, vol. 198, Academic Press, New York, NY, USA, 1999.

[36] R. Gorenflo and F. Mainardi, "Fractional calculus: integral and differential equations of fractional order," in Fractals and Fractional Calculus in Continuum Mechanics, Springer, Vienna, Austria, 1997.

[37] R. Hilfer, Applications of Fractional Calculus in Physics, World Scientific, Singapore, 2000.

[38] J. Ignaczak and M. Ostoja-Starzeweski, Thermoelasticity with Finite Speed, Oxford University Press, New York, NY, USA, 2010.

[39] L. Debnath and D. Bhatta, Integral Transforms and their Applications, Chapman \& Hall/CRC, Taylor \& Francis Group, London, UK, 2nd edition, 2007.

[40] H. M. Youssef, "Theory of fractional order generalized thermoelasticity," Journal of Heat Transfer, vol. 132, no. 6, pp. 1-7, 2010.

[41] F. Mainardi and R. Gorenflo, "On Mittag-Leffler-type functions in fractional evolution processes," Journal of Computational and Applied Mathematics, vol. 118, no. 1-2, pp. 283-299, 2000.

[42] A. Sur and M. Kanoria, "Fractional order generalized thermoelastic functionally graded solid with variable material properties," Journal of Solid Mechanics, vol. 6, no. 1, pp. 54-69, 2014.

[43] A. Sur and M. Kanoria, "Fractional heat conduction with finite wave speed in a thermo-visco-elastic spherical shell," Latin American Journal of Solids and Structures, vol. 11, no. 7, pp. 11321162, 2014.

[44] M. A. Ezzat and A. S. El-Karamany, "The uniqueness and reciprocity theorems for generalized thermoviscoelasticity for anisotropic media," Journal of Thermal Stresses, vol. 25, no. 6, pp. 507-522, 2002.

[45] M. A. Ezzat and A. S. El-Karamany, "On uniqueness and reciprocity theorems for generalized thermoviscoelasticity with thermal relaxation," Canadian Journal of Physics, vol. 81, no. 6, pp. 823-833, 2003.

[46] G. Jumarie, "Derivation and solutions of some fractional BlackScholes equations in coarse-grained space and time: application to Merton's optimal portfolio," Computers \& Mathematics with Applications, vol. 59, no. 3, pp. 1142-1164, 2010.

[47] A. S. El-Karamany and M. A. Ezzat, "Convolutional variational principle, reciprocal and uniqueness theorems in linear fractional two-temperature thermoelasticity," Journal of Thermal Stresses, vol. 34, no. 3, pp. 264-284, 2011.

[48] A. S. El-Karamany and M. A. Ezzat, "On fractional thermoelasticity," Mathematics and Mechanics of Solids, vol. 16, no. 3, pp. 334-346, 2011.

[49] A. Sur and M. Kanoria, "Fractional order two-temperature thermoelasticity with finite wave speed," Acta Mechanica, vol. 223, no. 12, pp. 2685-2701, 2012.

[50] S. Kothari and S. Mukhopadhyay, "A problem on elastic half space under fractional order theory of thermoelasticity," Journal of Thermal Stresses, vol. 34, no. 7, pp. 724-739, 2011.
[51] M. A. Ezzat and M. A. Fayik, "Fractional order theory of thermoelastic diffusion," Journal of Thermal Stresses, vol. 34, no. 8, pp. 851-872, 2011.

[52] A. E. Abouelregal, "Fractional order generalized thermopiezoelectric semi-infinite medium with temperaturedependent properties subjected to a ramp-type heating," Journal of Thermal Stresses, vol. 34, no. 11, pp. 1139-1155, 2011.

[53] F. Hamza, M. Abdou, and A. M. Abd El-Latief, "Generalized fractional thermoelasticity associated with two relaxation times," Journal of Thermal Stresses, vol. 37, no. 9, pp. 1080-1098, 2014.

[54] A. M. Zenkour and A. E. Abouelregal, "State-space approach for an infinite medium with a spherical cavity based upon twotemperature generalized thermoelasticity theory and fractional heat conduction," Zeitschrift für angewandte Mathematik und Physik, vol. 65, no. 1, pp. 149-164, 2014.

[55] R. B. Hetnarski and M. R. Eslami, Thermal Stressss-Advanced Theory and Application, Springer, 2008.

[56] M. R. Eslami, R. B. Hetnarski, J. Ignaczak, N. Noda, N. Sumi, and Y. Tanigawa, Theory of Elasticity and Thermal StressExplanatiions, Problems and Solutions, Springer Science + Business Media, Dordrecht, The Netherlands, 2013.

[57] J. Ignaczak and M. Ostoja-Starzewski, Thermoelasticity with Finite Wave Speeds, Oxford Science, 2010.

[58] R. Quintanilla, "A well-posed problem for the dual-phase-lag heat conduction," Journal of Thermal Stresses, vol. 31, no. 3, pp. 260-269, 2008.

[59] H. M. Youssef, "State-space approach on generalized thermoelasticity for an infinite material with a spherical cavity and variable thermal conductivity subjected to ramp-type heating," The Canadian Applied Mathematics Quarterly, vol. 13, no. 4, pp. 369-390, 2005.

[60] L. Y. Bahar and R. B. Hetnarski, "State space approach to thermoelasticity," in Proceeding of 6th Candian Congress of Applied Mechanics, pp. 17-18, University of British Columbia, Vancouver, BC, Canada, 1977.

[61] H. M. Youssef and E. A. Al-Lehaibi, "State-space approach of two-temperature generalized thermoelasticity of onedimensional problem," International Journal of Solids and Structures, vol. 44, no. 5, pp. 1550-1562, 2007. 


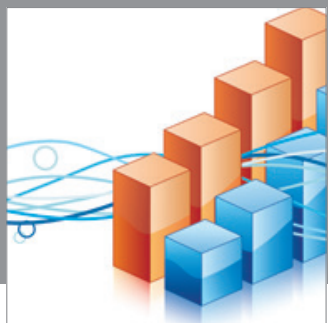

Advances in

Operations Research

mansans

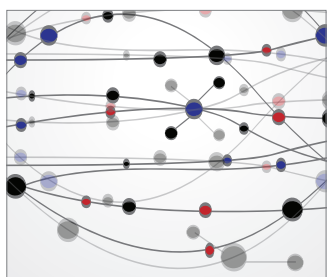

The Scientific World Journal
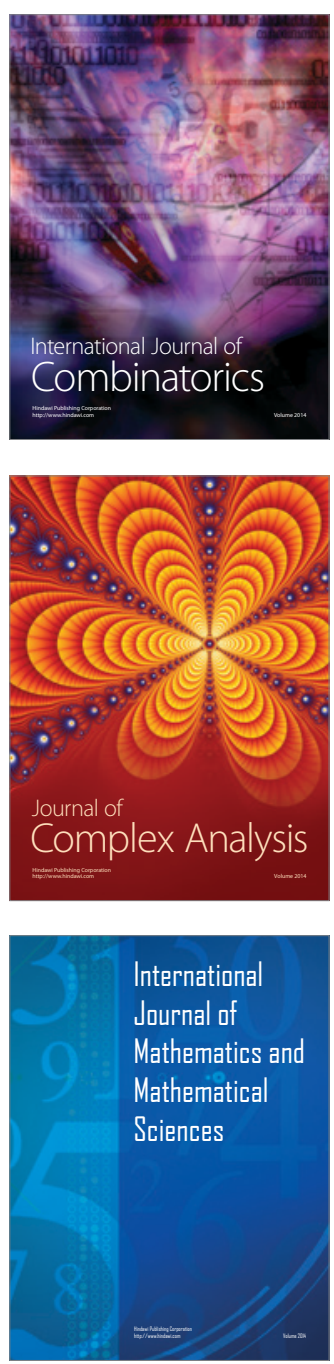
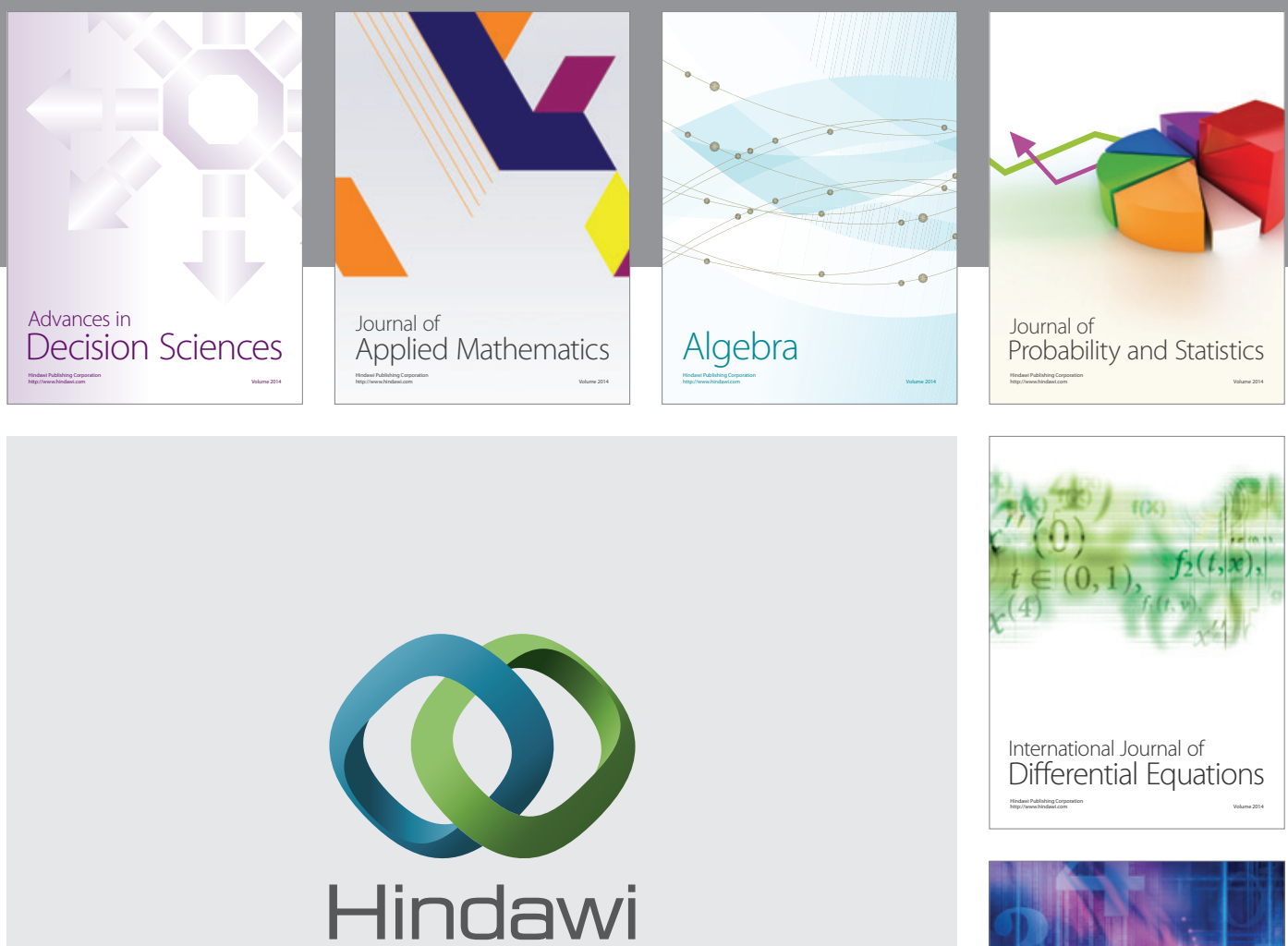

Submit your manuscripts at http://www.hindawi.com
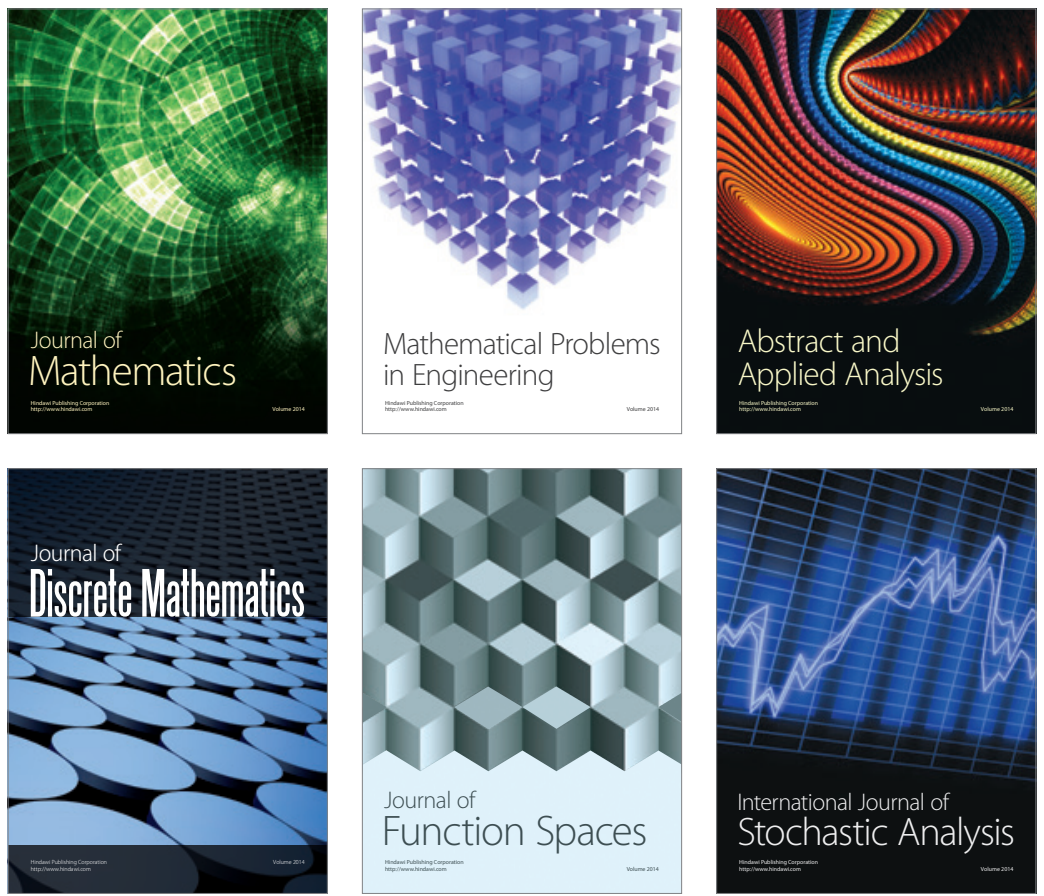

Journal of

Function Spaces

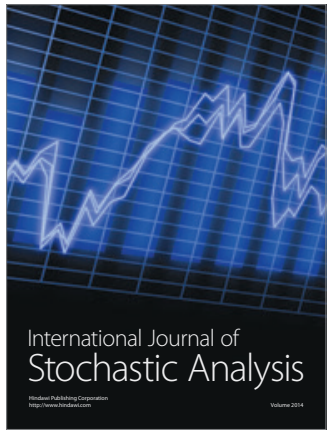

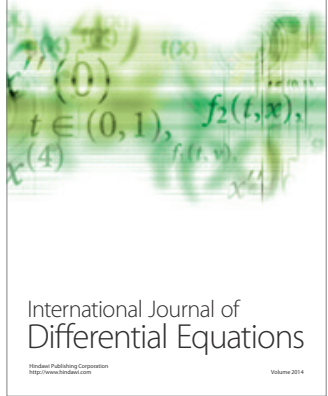
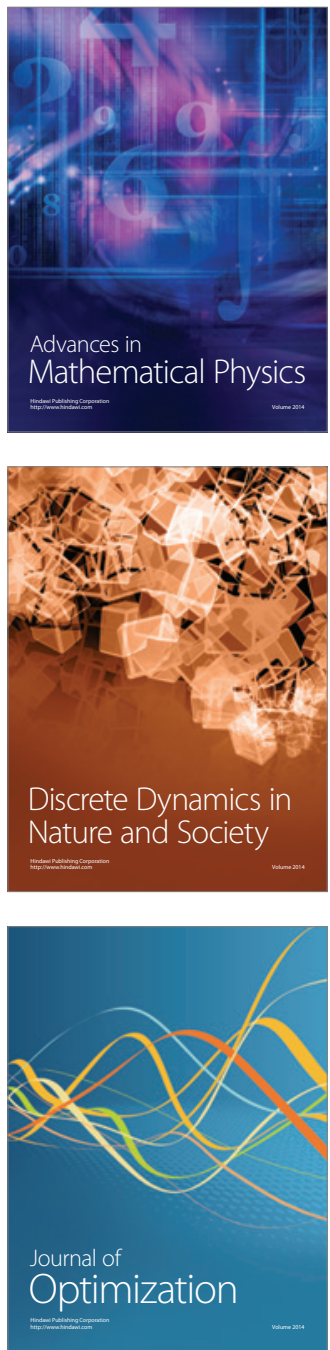